\title{
GESTIÓN ESTRATÉGICA DE LAS ÁREAS FUNCIONALES DE LA EMPRESA: UNA PERSPECTIVA COMPETITIVA INTERNACIONAL
}

\section{STRATEGIC MANAGEMENT ACROSS ENTERPRISE FUNCTIONS: AN INTERNATIONAL COMPETIVENESS PERSPECTIVE}

\author{
Luis Fernando Bustamante Zapata ${ }^{1}$ \\ Isabel Amalia Porto Pérez ${ }^{2}$ \\ Fernando Hernández Taboada ${ }^{3}$
}

\section{Resumen}

La internacionalización de las organizaciones aparece como una posibilidad de crecimiento empresarial o por lo menos una estratagema para la supervivencia en el mundo globalizado, altamente competitivo y sujeto a continuos y complejos cambios. El propósito de este documento es describir la gestión estratégica de las áreas funcionales de la empresa -técnica, comercial, financiera, contable, de seguridad y administrativa- como requisito para la competitividad internacional. Para hacerlo, se recurre al método analítico - sintético enunciando las presiones del entorno global que transforman las áreas funcionales de la empresa. Luego se plantean algunas de las posibles estrategias aplicables en cada área que facilitan la competitividad internacional de las organizaciones. Con base en los referentes teóricos se concluye que la gestión estratégica de las áreas de la empresa es una de las múltiples formas de desarrollar capacidades que faciliten la internacionalización organizacional. Es necesario ampliar el debate para continuamente completar las estrategias aplicables desde una perspectiva gerencial.

Palabras clave: Gestión estratégica, competitividad internacional, internacionalización, áreas funcionales de la empresa

Recibido: 30 de octubre de 2013
Aceptado: 20 de diciembre de 2013

Abstract

Internationalization is a real chance of growth, or at least an important survival strategy in a globalized, highly competitive and changing market. This document describes strategic management across enterprise functions technical, commercial, financial, accounting, security and management- as a required action for international competitiveness. We followed the analytical method listing the demands of a globalized market that transform every enterprise area. Then we propose some of the possible applicable strategies for each of pressure in order to acquire international competitiveness. Based on theoretical references, it is established that strategic management across enterprise functions eases organizational internationalization. It is necessary to further debate on applicable strategies from a managerial perspective.
Key words: Strategic management, international competitiveness, internationalization, enterprise functions.

\footnotetext{
1 Negociador Internacional, Magíster en Administración, Candidato a Doctor en Humanidades. Gerente Medellín, Comercializadora y Representaciones ANTIOTRADING S.A.S.E-mail: Ifbustamantez@gmail.com

2 Administradora de Empresas, Magíster en Administración. Subdirectora Administrativa y Financiera de la E.S.E. San Francisco de Asís de Sincelejo, Colombia.E-mail: isabel.porto@unisucre.edu.co

3 Ingeniero Agroindustrial, Magíster en Administración. Profesor Investigador, Facultad de Ingenierías. Universidad de Sucre, Colombia. E-mail:fernandodht@gmail.com
} 


\section{Introducción}

La competitividad constituye un referente obligado para aquellas empresas que desean aprovechar las diferentes oportunidades de negocios internacionales. Es una condición necesaria para la gestión global de las funciones esenciales de la empresa al interiorizar como objetivo la capacidad de penetrar en mercados mundiales (Toledo, 1997). Por tanto, las compañías deben orientarse a la creación de vínculos estables con diferentes actores a modo de estrategia de internacionalización (Galván, 2003).

En la era de la globalización la empresa debe competir siguiendo las presiones de altos estándares de calidad, compradores exigentes, entornos dinámicos y fuerzas tecnológicas de punta (Cullen \& Parboteeah, 2009). Además, los gobiernos continúan liberando los mercados y los convierten en protagonistas centrales de la actividad económica. Esto y el establecimiento de otras condiciones al interior y exterior de la empresa, resultarían en lo que se conoce como competitividad internacional (Porter, 2007).

El estudio de la competitividad ha ocupado gran parte de los esfuerzos de empresarios e investigadores, desde perspectivas interdisciplinarias como la política pública y la cultura (Bustamante, Arboleda, \& Gutiérrez, 2010). En general, la explicación se ha centrado en factores externos a la empresa; sin embargo, al comparar resultados de empresas de un mismo país o de un mismo sector, es posible encontrar diferencias lo que sugiere un estudio de factores endógenos.

Las decisiones de estrategia, diseño organizativo y asignación de recursos específicos, en la búsqueda de mejores condiciones de competencia en el entorno internacional, hacen responsables a las empresas de su relativo éxito (Acosta \& Medina, 1999). Desde esta perspectiva, la competitividad internacional presupone que la empresa pueda modificar sus condiciones de interacción y consolidación de resultados en los mercados. Por supuesto, existen muchas maneras de hacerlo y lo anterior puede estar orientado por la gestión estratégica de las funciones esenciales de la empresa, a lo cual se alude en este artículo.

Los mapas de competitividad (Rojas, 2002), redes de conceptos articulados que revelan el grado relativo de competitividad de la empresa y los campos susceptibles de mejoramiento, deben modificarse con el fin de conquistar, mantener y ampliar la participación en los mercados. De acuerdo con (Rialp \& Rialp, 2002), la competitividad internacional debe venir antes que la internacionalización. Por ende, el propósito de este documento es describir la gestión de las áreas funcionales para la competitividad internacional. Lo anterior es válido únicamente desde el nivel funcional de estrategia (Serna, 2008).

Para abordar entonces el problema resultante debido a las presiones o tendencias que la globalización genera y las transformaciones necesarias en términos de estrategia, se ahonda en los conceptos de competitividad y gestión estratégica internacional. Luego se presenta el método de acercamiento al trabajo para después describir los resultados. Finalmente, en las conclusiones, se abre un espacio de discusión haciendo un llamado a empresarios y académicos para que fortalezcan con su experiencia y nuevos enfoques los resultados presentados.

\section{Marco Teórico y Metodología}

\subsection{Marco Teórico}

\section{Competitividadinternacional}

La mayoría de definiciones y trabajos acerca de la competitividad, proviene de desarrollos a partir de teorías clásicas económicas que dieron forma al marco conceptual. Específicamente, la competitividad internacional comenzó a ser 
estudiada en el siglo XVII por las teorías de comercio internacional, las cuales se encuadran dentro de dos grandes líneas de pensamiento: universalista y particularista. Esto implica un manejo gerencial que logre articular todas las funciones de la empresa hacia el logro de los objetivos organizacionales, entendiendo la competitividad internacional como:

“... la capacidad de una empresa, industria, distrito industrial o localidad dentro de una misma nación para conservar o incrementar su participación de mercado en aquellos bienes o servicios cuya importancia en el comercio, consumo y valor agregado mundiales e interregionales esté creciendo y se espera que continúe haciéndolo en el futuro" (Berumen, 2006 p.61).

Las empresas que alcanzan los más altos niveles de competitividad internacional son las que logran eficiencia en cada una de las áreas funcionales por medio de aprendizaje organizacional y la constitución de factores claves de éxito de McKinsey -estrategia, sistemas, estructura, estilos, habilidades, personal- (Peters \& Waterman, 2004). En el contexto internacional, las presiones globales transforman éstas áreas, condicionando el desempeño a un nivel de desarrollo organizacional lo que para algunos posibilita el establecimiento de ventajas competitivas.

Aunque entrar en el debate de la competitividad no es el propósito de este artículo, es importante resaltar que múltiples aportes pretenden, además de definir el concepto de competitividad internacional, sentar las bases de los factores que propician dicho fenómeno. Según Lombana \& Rozas (2009), la Harvard Business School y el Institute of Management and Development (IMD), son referentes pioneros en el tema de competitividad. Ahora bien, el estado de la competitividad internacional del país ha sido estudiado por Porter et al. (2002), enunciando el papel que empresas, sectores, clústeres y el gobierno tienen en este aspecto. No obstante, el empresario para ser productivo, innovador y rentable requiere propuestas sobre las cuales tenga control en el manejo de su empresa en la internacionalización. Si bien el rol de las redes empresariales articuladas por el gobierno y otros entes es importante, es necesario volcar esfuerzos hacia el interior de las empresas retomando conceptos clásicos como las áreas funcionales de la empresa, vitales en la gerencia que Fayol (1974) definió como: técnica, comercial, financiera, contable, seguridad y administrativa.

\section{Gestión estratégica internacional}

La gestión estratégica internacional sigue el proceso estratégico tradicional y lo combina con el estudio de los negocios internacionales para la toma de seis decisiones en especial, sin obviar las demás que la empresa considere necesarias. Estas son: organización y estrategia internacional, análisis de oportunidades internacionales, selección y administración de modos de ingreso, administración de las operaciones internacionales, desarrollo y mercadeo de productos, y adquisición de recursos para los negocios (Wild, Wild, \& Han, 2001). Este proceso estratégico, que analiza variables endógenas y exógenas, como las fuerzas competitivas (Porter, 2008), puede verse graficado en la figura 1.

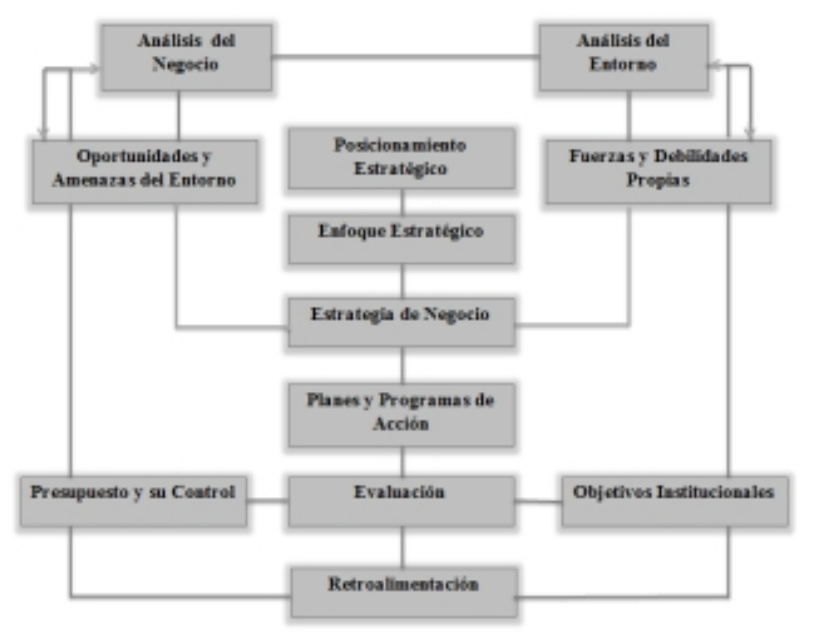

Figura 1. Proceso Estratégico (Haime, 2003) 
Aunque se retoman conceptos neoclásicos para la gestión estratégica internacional, estos son complementados hacia un entendimiento no programático de la estrategia (Morin, 1990), más flexible y en concordancia con el entorno competitivo. Al respecto Mintzberg (1987) alertó de la necesidad de pasar de una definición de estrategia como serie de caminos rígidos, a entenderla como cinco conceptos complementarios: planes, patrones, posición, maniobra y perspectiva. Así, cobran relevancia conceptos como la estrategia emergente y se revalúa la idea de que la estrategia descansa únicamente en la efectividad operacional (Porter, 1996).

De acuerdo con Wild, Wild, \& Han (2001) la principal diferencia entre la gestión estratégica tradicional y la internacionales que ésta última debe atender las presiones de múltiples ambientes nacionales de negocios. Además, Ghemawat (2010) soporta en la innovación la posibilidad de generar valor y de esa manera consolidar una perspectiva estratégica, ubicando al estratega internacional en la obligación de gestionar el conocimiento hacia la innovación.

\subsection{Metodología}

Para abordar el problema acerca de las potenciales estrategias que se pueden aplicar en las áreas funcionales de la empresa encaminadas hacia la competitividad internacional se parte de un recorrido documental. De acuerdo a lo anterior, se optó por el método analítico-sintético como lo define Feng Qi (Huang, 2002 p. 220). Este fue puesto en práctica siguiendo la propuesta de actividades que Miles \& Huberman (1994, p. 10) listan: recolección de datos, reducción de datos, presentación de datos y construcción y verificación de conclusiones.

La recolección de datos tuvo lugar entre junio y noviembre de 2011 bajo los parámetros de investigación documental descritos por Hoyos (2000), con los cuales se profundizó en cada una de las áreas funcionales de la empresa. Luego se redujeron los datos para organizar la información recolectada en concordancia con cada área funcional, identificando tendencias en el entorno global que deberían tener respuesta por medio de estrategias para la competitividad internacional.

Posteriormente, con base en el marco teórico, se describieron algunas de las posibles acciones utilizadas en las áreas funcionales de la empresa, que permiten desde esta óptica estratégica perfilar las organizaciones hacia mejores posiciones competitivas en el entorno global.

\section{Resultados y Discusión}

Ejercer la gerencia es conducir la empresa hacia su objeto tratando de sacar el mejor partido de todos los recursos disponibles (Sallenave, 2002), es asegurar la marcha de las funciones técnica, comercial, financiera, contable, de seguridad y administrativa. De esta manera se espera que la empresa desarrolle habilidades distintivas que se manifiesten en ventajas competitivas para superar a sus competidores (Hill \& Jones, 2000). Allí radican los aspectos sobre los cuales el gerente puede directamente influir.

En esencia, la definición de estrategia competitiva consiste en describir cómo la empresa va a competir, cuáles deben ser sus objetivos y qué políticas serán necesarias para alcanzar tal posición en el ámbito internacional (Deresky, 2005). Por supuesto una de las muchas maneras de hacerlo es transformando la estructura funcional. Lo anterior debe responder a la evaluación del ambiente nacional de los negocios (Wild, Wild, \& Han, 2001), es decir, al comportamiento de las variables ambientales, que dan origen a los siguientes fenómenos que impactan cada área funcional listados en la tabla 1.

Ahora bien, estas presiones implican retos para la estrategia y es cierto que la táctica está condicionada al tipo de estrategia genérica de 


\begin{tabular}{|c|c|}
\hline Área funcional & Presión global \\
\hline Técnica & Deslocalización de la producción, estándares globales de producción \\
\hline Comercial & $\begin{array}{l}\text { Caída de las barreras comerciales, convergencia de los gustos del } \\
\text { consumidor }\end{array}$ \\
\hline Financiera & Auge de los países emergentes y los mercados financieros internacionales \\
\hline Seguridad & Tecnologías de la información y la comunicación (TIC) \\
\hline Contabilidad & Buenas prácticas internacionales \\
\hline Administrativa & Cumplir una propuesta de valor articulando las diferentes áreas \\
\hline
\end{tabular}

Tabla 1. Algunas presiones globales que transforman las áreas funcionales

internacionalización usada, es decir: global, internacional, trasnacional y multidoméstica (Hill \& Jones, 2000). No obstante, es posible describir algunas estrategias orientadas a fortalecer las áreas funcionales de las empresas, como se ve en la tabla 2:

\begin{tabular}{ll}
\hline Área Funcional & \multicolumn{1}{c}{ Estrategia } \\
\hline Técnica & Personalización, estandarización o logística y buffers (Miltenburg, 2005) \\
Comercial & Diferenciación, liderazgo en costos y segmentación (Porter, 1997) \\
Financiera & Inversión, financiamiento y utilidades, diversificación del riesgo (Desai, 2008) \\
Seguridad & Gestión informática y tecnológica(Espinosa, 1997) \\
Contabilidad & Auditoría, control, cuadro de mando integral (Kaplan \& Norton, 2009) \\
Administrativa & Gerencia integral y estandarización o adaptación de prácticas (Sallenave, \\
& 2002) \\
\hline
\end{tabular}

Tabla 2. Algunas estrategias en las áreas funcionales para la competitividad internacional

\subsection{Funcional Técnica}

Elárea técnica empezó a ser un importante objeto de estudio dentro de la administración consolidándose como una herramienta competitiva (Díaz, 1999). De esta forma, y en oposición a la tendencia de décadas anteriores, se propone una orientación desde un enfoque sistémico, necesaria al considerar a las empresas como sistemas abiertos en continua interacción con el entorno internacional (Miltenburg, 2005).

Para el caso del área técnica, es importante señalar que la única forma en que la empresa se desarrolle y actúe en el entorno global es la generación de valor agregado (Porter, 2007). No basta con ser operacionalmente efectivo para hablar de estrategia, en realidad las acciones del área técnica deben estar orientadas a cumplir con una propuesta de valor diferenciadora, como se anota a continuación:
La estrategia de producción de bienes o procesos de servicios incluye planes de corto y largo plazo donde se compilan decisiones y políticas para el manejo de procesos, infraestructura, aprovisionamiento, inventarios, mantenimiento, modernización, talento humano, entre otros, que guían la consecución de los objetivos del área técnica, definidos y fijados en conformidad con la estrategia de la organización, con la finalidad de mejorar resultados y lograr una ventaja sobre los competidores que sea sostenible (Díaz, 1999).

Una propuesta de estrategia para el área funcional técnica, se encuentra en Avella, Fernández, \& Vázquez (1999), quienes describen dos posibilidades de producción asociadas a la fabricación centrada en la calidad con costos reducidos o la fabricación centrada en la entrega. La primera se enfoca en manufacturar una gama reducida de productos sin defectos, duraderos, con valor agregado en términos de calidad, flexibilidad y servicio. Para tal fin se debe emplear un sistema de prospectiva tecnológica, realizando cambios a precios razonables y respondiendo con rapidez a los requerimientos del cliente.

Una segunda estrategia sería manufacturar productos estandarizados en grandes volúmenes tratando de aprovechar la convergencia de los gustos del consumidor dadas por el avance de la globalización. Esto implica que la empresa debe dominar dos habilidades, la gestión de la calidad y la gestión de la tecnología, para acceder a los beneficios de las economías de escala y la curva de aprendizaje.

La gestión de la calidad, en este panorama, se entiende como una práctica administrativa que garantiza la idoneidad de los bienes y servicios ofrecidos y la eficiencia de los procesos y procedimientos administrativos empleados para obtenerlos, es el eje central de la construcción de una cultura de calidad. Por otro lado, la gestión tecnológica, actúa como socia en la reducción de costos, por medio de la aplicación de los 
principios administrativos a los procesos de desarrollo, adquisición, asimilación y uso de la tecnología para la producción de bienes y servicios (Román, 2004).

\section{2 Área funcional comercial}

La prosperidad de una organización depende tanto del área comercial como del área técnica; si no existen mecanismos para llevar los productos o servicios al mercado, la empresa arriesga su supervivencia. Saber comercializar es tan importante como saber desarrollar procesos productivos de bienes o servicios. (Fayol, 1974). En un entorno global, es preciso ajustarse a normas y estándares internacionales, especificaciones para acceder a nuevos mercados, y por supuesto a diferentes legislaciones comerciales, así como a las necesidades locales de cada producto y región.

Para el área comercial, las labores de mercadeo son vitales (Kotler \& Keller, 2006), ya que la competitividad está marcada por acciones de inteligencia de mercados, atendiendo a los gustos y preferencias del consumidor a través de una propuesta de valor. El área comercial precisa efectuar la venta de forma rentable y beneficiosa para la empresa, abarcando toda la cadena de valor y coordinando las actividades del equipo comercial con los servicios que intervienen antes y después de la venta.

Porter (1997) considera para la función comercial estrategias de liderazgo en costos, diferenciación y alta segmentación o enfoque. La estrategia de liderazgo en costos, se basa en priorizar la eficiencia en costos, por medio de la optimización de los procesos, el manejo de curva de experiencia y aprendizaje y el mejoramiento continuo, entre otras. La segunda implica el uso de diseños, marcas, tecnología, o manejo de la cadena de valor para, por medio de intangible, hacer el producto algo, especial en comparación con otros sustitutos y complementarios. Finalmente, la tercera, busca la satisfacción de un nicho específico de mercado por medio de la inversión constante en inteligencia comercial, gestión del conocimiento y manejo de las relaciones con el cliente. Requiere procesos de apoyo que ayuden a aclarar, recopilar, registrar y procesar información de los clientes en términos de estilo de vida, perfil comprador, hábitos comerciales, entre otros.

\section{3 Área Funcional Financiera}

Es necesario el acercamiento entre la estrategia competitiva, como guía para la asignación de recursos, y la búsqueda de posiciones favorables para financiar los gastos y costos de personal, inmuebles, materias primas, intangibles, etc. Se necesita una hábil gestión financiera para ubicar capitales y sacar el mejor partido posible de los eventos circunstanciales que se suscitan en la dinámica global; es una condición sine qua non, para poder competir en el ámbito internacional (Fayol, 1974).

Desde esta perspectiva, la competitividad presupone que las empresas tienen la capacidad de influir en la competencia y sus resultados en los mercados. Dicha capacidad motiva el cumplimiento de requisitos sobre los factores financieros (inversiones, gestión de recursos, financiación, entre otros). Sin embargo, dado un pensamiento estratégico, el análisis de la política financiera estatal, constituye también una posible fuente de ventaja competitiva o de refuerzo para las ya existentes (Acosta \& Medina, 1999).

El papel de las finanzas en la explicación de la competitividad internacional implica ventajas generadas en el correcto manejo de los activos y el flujo de caja. Así las cosas, el primer reto consiste en definir la estructura adecuada de capital de acuerdo con los riesgos del entorno, ahora compuesto por diferentes ambientes nacionales denegocios.

Las estrategias financieras constan de tres acciones principales: planeación, ejecución y 
control, todas de suma importancia para la supervivencia y consecución de los objetivos de la organización. Estas se manifiestan por medio de tres decisiones fundamentales (Besley \& Brigham, 2008): inversión, financiamiento y política de repartición de utilidades. También la gestión estratégica internacional de las finanzas trata decisiones como el financiamiento de las sucursales y filiales, la repatriación de capitales, el análisis de oportunidades de negocio y la integración de socios capitalistas (Desai, 2008).

Sobre la inversión y financiamiento de sucursales, es prudente establecer criterios conservadores, por cuanto en el plano internacional, la confianza financiera es llevada al límite. La problemática de la cartera internacional y la legislación comercial para la gestión de cobros es complicada (Cullen \& Parboteeah, 2009); a esta realidad se le suman los riesgos cambiarios. Esto obliga a la empresa a evaluar oportunidades de negocios en diferentes entornos y no sólo el análisis del valor presente de los flujos de dinero esperados. El criterio del valor presente neto y el presupuesto de capital como proyección de los flujos de operación netos de la inversión potencial (Besley \& Brigham, 2008), pasan a un segundo plano frente a modelos de gestión del valor económico agregado y gestión del riesgo por medio de estocásticos de simulación.

En el caso del establecimiento de sociedades o alianzas para financiar las operaciones, la estrategia deberá considerarse no sólo en correspondencia con el resultado económico que ésta sea capaz de lograr, sino con los típicos problemas de la cooperación competitiva; en tal sentido, cabe destacar que las estrategias al respecto apuntan directamente hacia el mayor o menor riesgo financiero de la empresa, por lo que en muchas ocasiones en la práctica se adoptan estrategias más o menos arriesgadas en dependencia del grado de aversión al riesgo de los inversionistas y administradores, o simplemente como consecuencia de acciones que conllevan al mayor o menor endeudamiento, o sea, no a priori o elaboradas, sino resultantes (Reyes, 2008).

En cuanto al movimiento de recursos entre casa matriz, filiales y sucursales, recomienda crear un equipo de profesionales financieros que se establezca en los diferentes ambientes nacionales, conociendo su legislación y práctica financiera para evaluar si es posible la transferencia de recursos y en tal caso definir las mejores acciones en términos cambiarios y financieros para aprovechar los créditos de las operaciones internacionales.

\section{4 Área Funcional de Seguridad}

Su misión es proteger los bienes y las personas contra todo obstáculo de orden social que pueda comprometer la marcha y hasta la vida de la empresa. Es, de manera general, toda medida que da seguridad a la empresa y tranquilidad al personal (Fayol, 1974). Hoy en día, la empresa internacional depende del uso de las tecnologías de información y la comunicación para la gestión estratégica de la seguridad. Estas pueden ser o no mediadas por sistemas automáticos o computarizados.

La seguridad influye notoriamente en la competitividad de las empresas ya que es importante para garantizar el desarrollo de una propuesta de valor, que contemple el mínimo riesgo a los grupos de interés de la organización. Sin embargo, para que esta función tenga los resultados que se desea, es importante el compromiso y esfuerzo de la gerencia por mejorar cada día más en los procesos, así como los recursos que se destinan para prevenir los factores de riesgo en la organización, en especial las que se desarrollan en el ámbito internacional.

Las presiones ejercidas por los gobiernos que imponen cada día regulaciones que restringen la acción empresarial, gremios que vigilan las 
prácticas organizacionales, clientes con acceso a gran cantidad de información y trabajadores calificados y con posibilidad de movilidad internacional, continúan obligando a la empresa a mejorar para mantener buenas relaciones con los actores del ambiente internacional de negocios (Wild, Wild, \&Han, 2001).

La gestión estratégica en el área de seguridad, precisa de sistemas, ejecución, inspección, y realimentación que aseguren la integridad de personas, procesos y recursos, entre otros. El sistema debe permitir detectar con prontitud cualquier problema que pudiese afectar la seguridad e integridad de los mismos. Para tal efecto, se desarrollan actividades de gestión informática y tecnológica enfocadas en identificar factores de riesgos y lograr que ante cualquier eventualidad, el impacto tanto a las personas como a las instalaciones sea mínimo, esto es parte del Ilamado proceso de aprendizaje organizacional (Senge, 2004). Lo anterior comprende el establecimiento de un sistema estratégico de información, que registre, evalúe y prevenga todo tipo de riesgos en la empresa (Espinosa, 1997).

El área funcional de seguridad también debe encargarse de proteger los activos intangibles de la empresa como lo son, la información, las bases de datos, las marcas, la imagen corporativa, el know-how, entre otros (Stackpole \& Oksendahl, 2010). El auge de las TIC implica nuevas amenazas como los ataques cibernéticos, el robo de información, el atentado contra servidores e infraestructura informática, diferentes tipos de virus, espionaje industrial, y otros riesgos por los cuales se debe estar en alerta para su prevención y manejo. Algunas acciones para evitar estos impases incluyen, personal de vigilancia, desarrollo propio de software, tercerización de servicios, contratación de evaluadores de la robustez del sistema, y más importante aún, pensamiento estratégico de la cultura de una compañía o valor corporativo orientado a la seguridad de la empresa en todo sentido.

\section{5 Área Funcional de Contabilidad}

Es el órgano de visión y registro histórico de los resultados de la empresa. Debe permitir conocer en todo instante la situación de la empresa y la perspectiva de cumplimiento de objetivos. Una contabilidad sencilla y clara permite tener una idea exacta de las condiciones de la empresa, lo cual la convierte en un medio poderoso de dirección (Fayol, 1974). Sin embargo, por diferentes razones culturales, institucionales y técnicas, la empresa internacional enfrenta el reto de armonizar su contabilidad global dada la gran variedad de prácticas contables (Berry, 1987). Sin el apego a buenas prácticas internacionales de contabilidad, será difícil consolidar estados financieros, evaluar el estado de la empresa, acceder a recursos monetarios y responder a los diferentes sistemas legales.

Por lo tanto, la contabilidad en la gestión estratégica internacional debe establecer las causas de la rentabilidad de las distintas industrias y considerar cómo las unidades de negocios, con diferentes estrategias, sobreviven en un entorno competitivo (Porter, 1997). Además, para el área de contabilidad avanza la adopción de las Ilamadas Normas Internacionales de Información Financiera, NIIF, que obligará a las empresas a unificar el reporte de su contabilidad. Esto puede ser una salida a los problemas de traducción de los informes y la consolidación de estados financieros de las casas matrices, sucursales y filiales.

La contabilidad es de gran importancia en las empresas, y más aún si están con la intención de internacionalizarse, especialmente, por la información que brinda para la toma de decisiones en los diferentes niveles de la organización y en un sentido amplio por los grupos de interés, quienes requieren de ésta para facilitar sus relaciones con el ente económico. Procurando en todo caso la satisfacción de las necesidades de información, la contabilidad debe diseñarse teniendo en cuenta las prácticas de 
cada actividad. Tales como la naturaleza de sus operaciones, las NIIF y los aspectos puntuales del ambiente nacional de negocios. Desai (2008, p. 112) sustenta que "es necesario codificar las prioridades y prácticas que se pueden adaptar a las condiciones locales" siempre y cuando se guarden criterios de decisión que beneficien tanto a la casa matriz como a las filiales y sucursales.

De acuerdo con Johnson \& Kaplan (1995), los componentes principales para la competitividad internacional incluyen relaciones a largo plazo con diferentes grupos de interés, flexibilidad en los procesos, eliminación de restricciones y enfoque hacia la información contable. Estos autores exponen que los sistemas efectivos de control administrativo deben diseñarse de arriba hacia abajo; señalan que es vital para las empresas contar con sistemas de información que liguen sus acciones con los mandatos de la competencia global. $Y$ eso incluye costeo basado en actividades, análisis de la rentabilidad del cliente, gestión del valor económico agregado, sistemas empresariales de planeación de recursos, costeo justo a tiempo, tableros de comando, aplicación de la teoría de las restricciones y asignación de costos por objetivos.

Otro importante factor global que afecta al área de contabilidad, es el tema de impuestos. Cada ambiente nacional de negocios impone pagos de varios tipos relacionados con la actividad empresarial. Por lo tanto, el manejo oportuno de la contabilidad, la ubicación en áreas con exenciones arancelarias, cambiarias y tributarias, los tratados que evitan la doble tributación, las figuras jurídicas con incentivos tributarios y otros movimientos son tareas de la estrategia de contabilidad.

Es claro que la auditoría, el control, y la utilización de herramientas como el cuadro de mando integral para articular todas las perspectivas estratégicas de la empresa (Kaplan \& Norton, 2009), descargan en la contabilidad las necesidades de información que el gerente internacional requiere para realizar una gestión estratégica de las actividades empresariales.

\section{6 Área Funcional Administrativa}

Tiene como tarea la previsión, organización, coordinación y control; donde prever, es escrutar el futuro y articular el programa de acción; organizar, es constituir el doble organismo, material y social, de la empresa; dirigir, es hacer funcionar el personal; coordinar, es relacionar, unir, armonizar todos los actos y todos los esfuerzos; controlar, es procurar que todo se desarrolle de acuerdo con las reglas establecidas y las órdenes dadas (Fayol, 1974).

Bajo la óptica actual, la gran mayoría de autores ha obviado la función de coordinación, incluyéndola en la dirección. Incluso la teoría neoclásica de Peter Drucker (1964), la omite, conformando las famosas máximas de la administración moderna: planeación, organización, dirección y control. Tal y como expone Fayol (1974) el área administrativa sincroniza y coordina las demás por medio del proceso listado. Desde una mirada global, las funciones de ésta área constituyen un desafío para la gerencia, dada la complejidad de articular tantos esfuerzos, la dinámica organizativa, y sobre todo la necesidad de contemplar el escenario competitivo internacional.

La gestión estratégica de las funciones administrativas descansa en la estandarización o adaptación de prácticas administrativas. Sobre el particular, Peters \& Waterman (2004) afirman que la estrategia depende de siete factores: objetivos estratégicos, estructura, habilidades, estilos, cultura organizacional, personas, y sistemas. En armonía con el pensamiento de Fayol, por ejemplo, en la actividad de previsión, el factor clave es la definición de patrones a seguir, esto implica definir objetivos haciendo uso de la planeación estratégica, para responder de manera efectiva a retos inciertos como los que plantea la globalización. 
Se requieren mecanismos para obtener el logro de objetivos estratégicos que tributen a la permanencia y la competitividad internacional de las organizaciones, esto significa que las metas estén en línea con los objetivos a largo plazo, condicionados por el compromiso de ejecutar las actividades definidas, todo lo cual es controlado por indicadores. Una herramienta para el análisis de estas operaciones son los planes estratégicos, que se convierten en carta de navegación de la organización utilizando técnicas que establezcan y prioricen los aspectos más relevantes de la estrategia a ejecutar para mejorar la competitividad.

El segundo elemento es la estructura, la cual señala las particularidades sobre los aspectos básicos del proceso vinculado con la estructura administrativa de las organizaciones, las relaciones de autoridad y las responsabilidades administrativas inherentes para el desarrollo de las actividades, es decir, su proceso organizativo. Sobre este proceso se sugiere la constitución de organizaciones tipo red, que propician la existencia de equipos que se auto-organizan, la coexistencia de una estructura jerárquica con organizaciones reticulares informales, abiertas, no jerarquizadas, y directivos adaptados a las tendencias globales.

En la dirección se incorporan varios factores como: los estilos, las habilidades, las personas y la cultura organizacional, por algo su carácter interpersonal; de allí su complejidad. La estrategia debe estar orientada a integrar el estilo de dirección participativo en los sistemas de administración, sustentados en cuatro variables (proceso decisorio, sistema de comunicaciones, relaciones interpersonales y los sistemas de recompensas y sanciones). Este sistema se refiere a la necesidad de empoderar a los colaboradores, es decir, es un estilo basado en la motivación, el liderazgo y la comunicación, en el cual se conjuga la dirección por objetivos, la dirección basada en valores, y la respuesta a las tendencias globales (Likert, 1969).
Se trata de una nueva propuesta de dirección integral con muchos matices gerenciales de carácter humanista e integrador, donde la persona se ubica en el centro del pensamiento administrativo para responder al contexto de competitividad creciente que exige altos niveles de rendimiento, confianza, compromiso, pertenencia y creatividad de parte de todas las personas que componen la empresa.

En lo que respecta al control, se puede establecer una estrategia sustentada en métodos de seguimiento continuo al desarrollo de los planes y la práctica del control múltiple en términos preventivos, concurrentes y de retroalimentación, es decir: antes, durante y después, en todos los procesos. Para ello la organización puede apelar a cualquier herramienta de control, como por ejemplo el Cuadro de Mando Integral (Kaplan \& Norton, 2009). El tablero de control debe incorporar control directivo estratégico, operativo y las aplicaciones existentes de medición en entornos dinámicos cuando la incertidumbre obliga a ser flexibles y administrar bajo escenarios más complejos que en el ámbito nacional.

\section{Conclusiones}

Los entornos competitivos generan presiones que transforman las estructuras de las organizaciones, especialmente, la globalización forma nuevos retos de los cuales ninguna empresa puede escapar (Deresky, 2005). Es por tales razones que la empresa requiere superar las etapas de diagnóstico y proponer mecanismos para potenciar su competitividad. Los nuevos mecanismos de gestión estratégica de la empresa, si bien se enmarcan en la acción de diferentes actores y fuerzas del entorno, deben volcar sus esfuerzos hacia elementos sobre los cuales el gerente tenga directa influencia. Estos por supuesto residen en su empresa de la cual es responsable, representante y líder. La gestión estratégica de las áreas funcionales de la empresa, constituye un elemento fundamental a la hora de competir en cualquier mercado; ya que puede 
fortalecer procesos al interior de la empresa. Es un llamado a reconsiderar las propuestas de la escuela estructuralista de la administración (Mintzberg, Ahlstrand, \& Lampel, 1998), como primer momento en el desarrollo de la internacionalización organizacional.

Futuros desarrollos deberán considerar un espectro de tendencias más amplio para proponer una gestión estratégica en concordancia con los factores del contexto. Aunque otras teorías organizacionales consideran al enfoque de áreas funcionales reduccionista, la necesidad de atender las afujias de la empresa Colombiana obligan a retomar perspectivas rápidamente replicables. De esta manera, los cambios en el ambiente nacional de negocios se deberán ver reflejados en ajustes en todos o muchos de los procesos, probable tema para estudio posterior.

Finalmente, es necesario desarrollar investigaciones sobre la gestión estratégica de las áreas funcionales de forma particular, tomando cada una por separado con el propósito de ahondar en las posibles acciones relacionadas con la competitividad internacional. También sobre la articulación de la información de los múltiples ambientes nacionales de negocios, para construir escalas de prioridades que satisfagan a la empresa internacional, sus socias, filiales, sucursales y por supuesto, el resto de grupos de interés.

\section{Referencias}

Acosta, M., \& Medina, U. (1999). Función Financiera y Estrategia Competitiva de la Empresa. Investigaciones Europeas de Dirección y Economía de la Empresa, 5(2), 55-68.

Avella, L., Fernández, E., \& Vázquez, C. (1999). Análisis de las estrategias de fabricación como factor explicativo de la competitividad de la gran empresa industrial española. Cuadernos de Economía y Dirección de la Empresa, 4, 235-258.
Berry, J. (1987). The need to classify worldwide accountancy practices. Accountancy, 100(1130), 90-91.

Berumen, S. (2006). Competitividad y desarrollo local. Madrid:ESIC.

Besley, S., \& Brigham, E. (2008). Fundamentos de Administración Financiera. México: Cengage Learning.

Bustamante, L., Arboleda, A., \& Gutiérrez, J. (2010). Cultura, política industrial y competitividad internacional de PyMEs en Colombia. Revista AdMinister, enero-junio(16), 83-98.

Cullen, J., \& Parboteeah, K. (2009). International Business: Strategy and the Multinational Company. New York: Routledge.

Deresky, H. (2005). International Management. Managing across Borders and Cultures. New Jersey: Prentice Hall.

Desai, M. (2008). The Finance Function in a Global Corporation. Harvard Business Review, JulyAugust, 108-112.

Díaz, E. (1999). La estrategia de producción como estrategia funcional: Una propuesta.Madrid:Tesis Doctoral.

Drucker, P. (1964). Managing for Results. New York: Harper \& Row.

Espinosa, Á. (1997). Planeación de sistemas estratégicos de información: Una visión cibernética. En Á. Galvis, \& Á. Espinosa, Estrategia competitividad e informática (pp. 193-208). Bogotá:Uniandes

Fayol, H. (1974). Administración Industrial y General. México: Herrero Hnos. Svcs. S.A. 
Galván, I. (2003). La formación de la estrategia de selección de mercados exteriores en el proceso de internacionalización de las empresas. Universidad de Las Palmas de Gran Canaria: Tesis doctoral. Recuperado el 25 de Agosto de 2009, de http://www.eumed.net/tesis/igs/.

Ghemawat, P. (2010). Finding your strategy in the new landscape. Harvard Business Review, 88 (2), 54-60.

Haime, L. (2003). Planeación financiera en la empresa moderna. México:ISEF.

Hill, C., \& Jones, G. (2000). Administración estratégica: un enfoque integrado. México: McGraw-Hill.

Hoyos, C. (2000). Un modelo para investigación documental. Guía teórico-práctica sobre construcciones de estados del arte.Medellín: Señal Editora.

Huang, Y. (2002). Feng Qi's ameliorism: Between relativism and absolutism. En N. Bunnin, \& C. Zhonggying (Edits.), Contemporary Chinese Philosophy (págs. 213-234). Oxford: Blackwell Publishers.

Johnson, T., \& Kaplan, R. (1995). Relevance Lost. Boston: Harvard Business School Press.

Kaplan, R., \& Norton, D. (2009). Mastering the Management System. Harvard Business Review, January, 63-77.

Kotler, P., \& Keller, K. (2006). Dirección de Marketing. México: Prentice-Hall.

Likert, R. (1969). Un nuevo método de gestión y dirección. Bilbao: Deusto.

Lombana, J., \& Rozas, S. (2009). Marco analítico de la competitividad: Fundamentos para el estudio de la competitividad regional. Pensamiento \& Gestión, 26, 1-38.

Miles, M., \& Huberman, M. (1994). Qualitative data analysis: An expanded source book. Thousand Oaks: Sage Publications.

Miltenburg, J. (2005). Manufacturing Strategy: How to Formulate and Implement a Winning Plan. Portland:Productivity Press.

Mintzberg, H. (1987). The Strategy Concept I: Five Ps For Strategy. California Management Review, $30(1), 11-24$

Mintzberg, H., Ahlstrand, B., \& Lampel, J. (1998). Strategy Safari: A guided tour through the wilds of strategic management. New York:The Free Press.

Morin, E. (1990). Introduction à la pensée complexe. Paris: ESPF Éditeur.

Peters, T., \& Waterman, R. (2004). In Search of Excellence: Lessons from America's Best-Run Companies. New York: HarperBusiness.

Porter, M. (1996). What is strategy? Harvard Business Review, 74(6), 61-78.

Porter, M. (1997). La Ventaja Competitiva. México: Cecsa.

Porter, M. (2007). La Ventaja Competitiva de las Naciones. Harvard Business Review (Edición América Latina), 85(11), 69-95.

Porter, M. (2008). The Five Competitive Forces That Shape Strategy. Harvard Business Review, January, 78-93.

Porter, M., Emmons, W., Brenes, E., Montenegro, S., Madrigal, K., Niño, L., \& Gisbert, R. (2002). Institutions for Competitiveness in Colombia. Bogotá: Andean Competitiveness Project. 
Reyes, M. (2008). Las estrategias financieras de las empresas. La Habana: Universidad de la Habana.

Rialp, A., \& Rialp, J. (2002). Estrategia internacional de la empresa. Barcelona:UOC.

Rojas, G. (2002). Conceptualización y métodos para la competitividad internacional. Cali: Universidad Santiago de Cali.

Román, R. (2004). Teoría y mediciones de competitividad: Una perspectiva. Bogotá: Universidad Distrital Francisco José de Caldas.

Sallenave, J. (2002). La Gerencia Integral. Bogotá: Editorial Norma.

Senge, P. (2004). La quinta disciplina: El arte y la práctica de la organización abierta al aprendizaje. Barcelona: Granica.
Serna, H. (2008). Gerencia Estratégica. Teoría Metodología- Alineamiento, implementación y mapas estratégicos.Bogotá:3R Editores.

Stackpole, B., \& Oksendahl, E. (2010). Security Strategy: From Requirements to Reality.Boston: Taylor \& Francis Group.

Toledo, F. (1997). Competitividad internacional y estratégica de las empresas colombianas. Gestión, $3,1-23$.

Wild, J., Wild, K., \& Han, J. (2001). International Business: An Integrated Approach. New Jersey: Prentice Hall. 